\title{
Modeling an Activation of Heart Ventricular Segments
}

\author{
Radovan Smisek ${ }^{1,2}$, Pavel Jurak ${ }^{2}$, Josef Halamek ${ }^{2}$, Filip Plesinger ${ }^{2}$, Ivo Viscor ${ }^{2}$, Magdalena \\ Matejkova $^{3}$, Pavel Leinveber ${ }^{3}$ \\ ${ }^{1}$ Brno University of Technology, Brno, CZ \\ ${ }^{2}$ The Czech Academy of Sciences, Institute of Scientific Instruments, Brno, CZ \\ ${ }^{3}$ International Clinical Research Center, St. Anne's University Hospital, Brno, CZ
}

\begin{abstract}
Background: Here, we present a $2 D$ activation model evaluating the activation of specific myocardium segments (MS). Activation times of specific MS can help to identify conduction disturbances which may result in more targeted treatment of patients.

Methods: 12-lead ECG signal was measured with a $5 \mathrm{kHz}$ sampling frequency. A total of 10 left bundle branch block (LBBB) and 5 right bundle branch block (RBBB) recordings were analyzed. The analysis includes the following steps: 1) QRS complexes detection and morphology clustering, 2) averaging of 150-1000 Hz envelopes of QRS complexes with dominant morphology, 3) a genetic algorithm (GA) produces artificial envelopes from (initially random) timing of MS activation. The task for GA is to produce envelopes the most similar to measured; then final timing reflects real activation of MS.

Results: Presented model determined activation of left ventricular $(L V)$ segments before right ventricular $(R V)$ segments in all RBBB patients (mean $61.4 \pm 13.7 \mathrm{~ms}$ ) and activation of $R V$ segments before the $L V$ segments in all $L B B B$ patients (mean $87.8 \pm 15.8 \mathrm{~ms}$ ). Computed activation of MS corresponds to the expected activation.

Conclusion: We introduced a new method determining activation times of MS; this is achieved non-invasively using only ultra-high-frequency precordial ECG signal.
\end{abstract}

\section{Introduction}

Determination of ventricular activation times from signals sensed on the body surface is a frequently discussed problem in recent years $[1,2,3]$. This issue is non-invasively solved by electrocardiographic imaging (ECGI) [1] technique with the inverse reconstruction of activation times on the epicardium. Current ECGI methods allow the determination of activation times with high resolution. However, it is necessary to sense signals from many electrodes on the patient's torso (often over 100 electrodes) $[1,4]$.
The ultra-high-frequency (UHF) ECG method and principles were first introduced in [5]. This article describes a method for determining activation times of 11 myocardium segments: 3 in the right ventricle, 3 in the left ventricle, 3 in the septum, and 2 in the apex. The ECG records were sense with only 6 standard precordial leads. ECG records were lacquered with a sampling frequency of $5 \mathrm{kHz}$ and 24-bit resolution [6]. Magnetic resonance imaging was also performed for each of the analyzed subjects to determine heart geometry. A total of 10 subjects with LBBB and 5 subjects with RBBB were tested.

\section{Method}

The block diagram of the proposed algorithm is shown in Figure 1. The presented method is based on GA. The inputs to this algorithm are heart geometry and amplitude envelopes measured from 6 precordial leads V1-V6. The genetic algorithm aims to determine the timing of MS activation that will generate amplitude envelopes as close as possible to the measured amplitude envelopes.

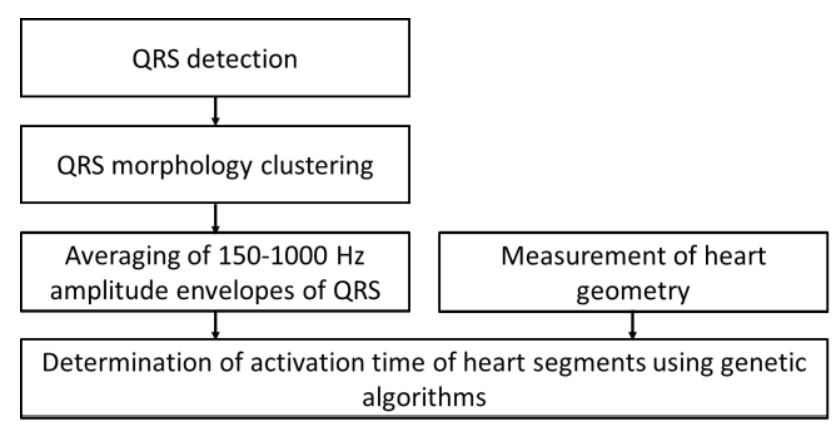

Figure 1. Block diagram of the proposed algorithm

\subsection{Detection and morphology clustering of QRS complexes}

The robust multichannel QRS detector [7] was used for QRS complex detection. The presented method uses ultra-high-frequency components of the QRS complex. 
These components are very weak. Averaging several QRS complexes will suppress noise and allow the analysis of these weak UHF components. This is necessary to divide the detected QRS complexes into morphological groups before averaging so that the average is not influenced by significantly noisy QRS complexes or pathologies, e.g. premature ventricular complexes. The algorithm used for morphology clustering is described in [8].

\subsection{Calculation of envelopes}

Amplitude envelopes were computed in frequency bands from $150 \mathrm{~Hz}$ to $1000 \mathrm{~Hz}$. It was computed for all QRS complexes with dominant morphology in all precordial leads. The amplitude envelopes of QRS complexes were then averaged. This averaged envelope is the input to the GA.

\subsection{Measurement of heart geometry}

The magnetic resonance scan was performed for each of the analyzed subjects to determine heart geometry. The heart ventricles were divided into 11 segments - 3 in the right ventricle, 3 in the left ventricle, 3 in the septum and 2 in the apex (blue rectangles in Figure 2). The distance of the center of each segment (black dots in Figure 2) from each sensing electrodes (red dots in Figure 2) and the size of each segment were manually measured. These values are necessary to subsequently generation of amplitude envelopes from the specified activation times of each segment.

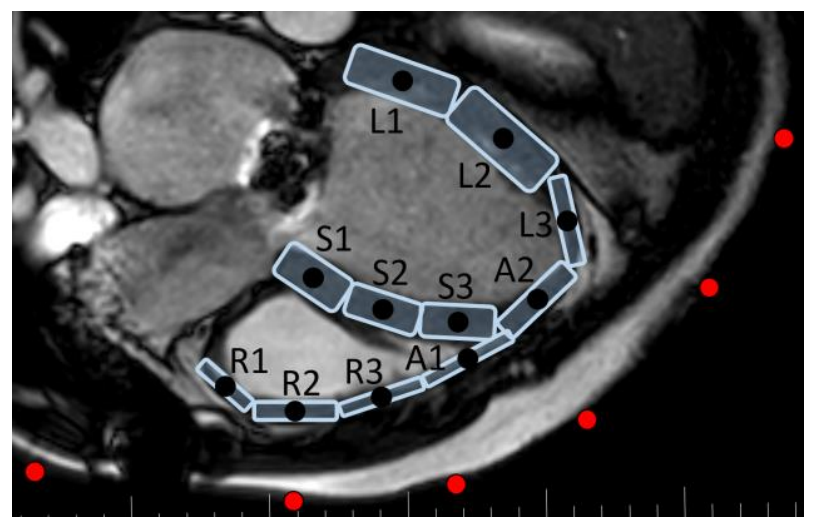

Figure 2. Myocardium segments (blue rectangles) and electrodes (red dots) in magnetic resonance scan of the heart

\subsection{Determination of activation times of myocardium segments}

The determination of activation times of ventricular segments was performed using GA. Each individual in the GA population represents one realization of the activation timing of all heart segments.

One individual in the GA population is represented by an $11 \times 48$ matrix. Each row of the matrix represents one heart segment and, each column is a time point at which we determine the activation of the segment. The distance of two adjacent time points is $5 \mathrm{~ms}$, so we analyze a total of $240 \mathrm{~ms}$.

The size of the GA population is 60 individuals. The initial population consists of random individuals.

The quality of each GA individual is evaluated according to the degree of similarity between the measured and generated amplitude envelopes.

Amplitude envelopes are generated for each lead according to the formula:

$$
y(t)=\sum_{i=1}^{11} \frac{S S(i) \times A c(t, i)}{S E(i)^{2}}
$$

where $y(t)$ is the value of the envelope amplitude at time $t, \operatorname{SS}(\mathrm{i})$ is the size of the segment $\mathrm{i}, \mathrm{Ac}(\mathrm{t}, \mathrm{i})$ is the value of activation of the segment $i$ at the time $t$, and SE(i) is the distance between the heart segment and the electrode sensing the currently calculated lead. Thus, Ac is a matrix described above optimized by a GA.

The creation of a new population (reproduction) is performed after the assignment of quality to each individual. The algorithm uses the principle of elitism. The two best individuals are automatically in the new population. The other offsprings are generated by randomly selecting pairs of individuals from the old population, from which three offsprings are generated according to the following formulas:

$$
\begin{aligned}
& \text { offspring } 1=0.5 \times \text { parent } 1+0.5 \times \text { parent } 2, \\
& \text { offspring } 2=1.5 \times \text { parent } 1-0.5 \times \text { parent } 2, \\
& \text { offspring } 3=-0.5 \times \text { parent } 1+1.5 \times \text { parent } 2 .
\end{aligned}
$$

The worst of the generated offsprings is deleted and the other two are part of the new population.

Single-point and three-point mutations occur further in new individuals. A single point mutation means that each value of each individual can be increased or decreased by 0.1 , with a probability of $10 \%$. The three-point mutation means that three consecutive values of heart activation in one heart segment are set to zero. Thus, this mutation ensures faster optimization. The multipoint mutation occurs in each offspring with a probability of $20 \%$.

The GA is stopped after 5 million iterations.

The output of the genetic algorithm is the distribution of activation in each segment. It is sometimes necessary to determine a one-time point of maximum activation of individual segments. This activation is determined at a center of gravity of the curve. 


\section{Results and discussion}

The results of the proposed algorithm were tested on the fifteen records, of which 10 were $\mathrm{LBBB}$ and 5 were RBBB.

Presented activation model determined activation of the left ventricular segments before the right ventricular segments in all RBBB patients (mean $61.4 \pm 13.7 \mathrm{~ms}$ ) and activation of the right ventricular segments before the left ventricle segments in all LBBB patients (mean $87.8 \pm$ $15.8 \mathrm{~ms}$ ). The calculated activation of the segments corresponds to the expected activation in $\mathrm{LBBB}$ and RBBB.

We do not have data for which the activation times are known. The results will be further shown in selected cases - one LBBB and one RBBB.

Determination of segment activation in a patient with LBBB is shown in a record with a QRS complex duration of $182 \mathrm{~ms}$ with typical LBBB morphology - qS in V1 and V2; slur or notch in I, aVL, V2, V5, and V6. In total, 379 QRS complexes of dominant morphology were detected.

Determination of segment activation in a patient with $\mathrm{RBBB}$ is shown in a record with a $\mathrm{QRS}$ complex duration of $184 \mathrm{~ms}$ with typical RBBB morphology - rSR' with negative T wave in V1 and qRS in I and V6. In total, 275 QRS complexes of dominant morphology were detected.

The envelopes calculated from the measured data and the corresponding envelopes generated by the segment activation timing selected by GA are shown in Figure 3 and Figure 4.

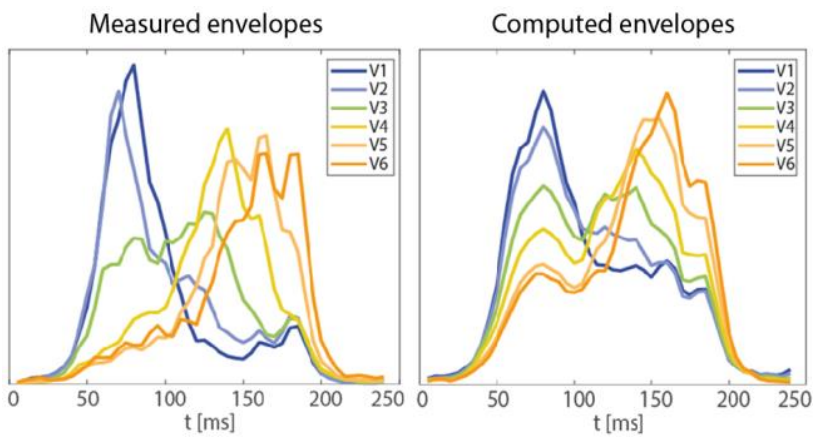

Figure 3. Envelopes of LBBB patient; envelopes generated from determined activation times of heart segments (right) and envelopes of the measured QRS complex (left)
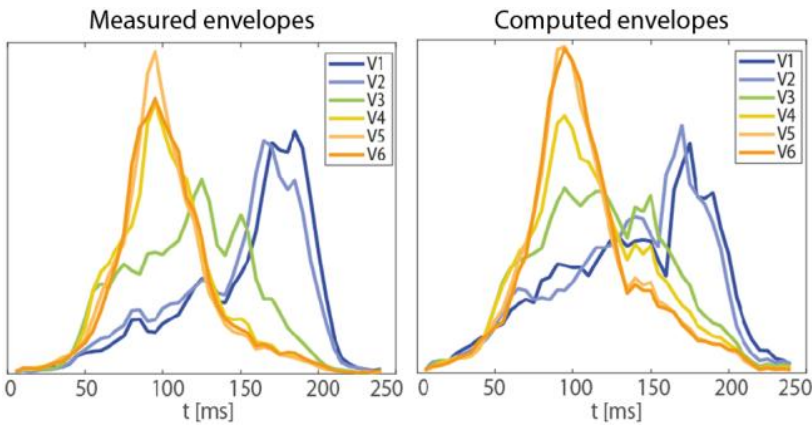

Figure 4. Envelopes of RBBB patient; envelopes generated from determined activation times of heart segments (right) and envelopes of the measured QRS complex (left).

It can be seen that the chronological sequence of extremes in individual leads remains the same. The biggest inaccuracies are that our method does not allow to generate activity only in the left ventricle sensing electrodes with minimal activity in other electrodes and vice versa. Higher than measured amplitudes are also generated in the leads sensing mainly the left ventricle at the time when the right ventricle is activated and vice versa.

Signals showing the activation of all segments are shown in Figure 5 and Figure 6.

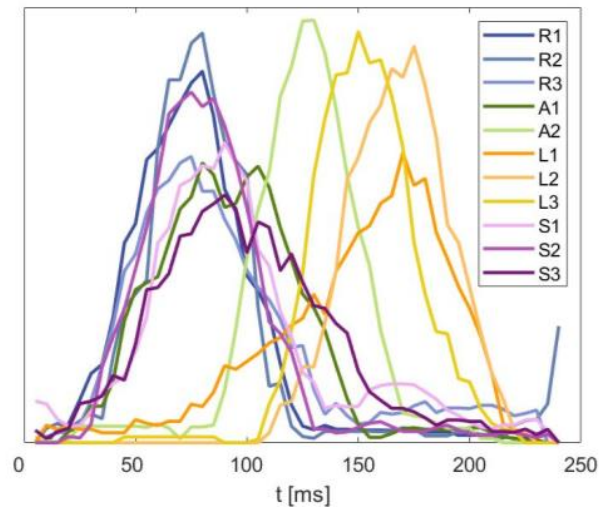

Figure 5. Activation of individual segments over time; LBBB record (the positions of the segments in the heart ventricle are shown in Figure 2). 


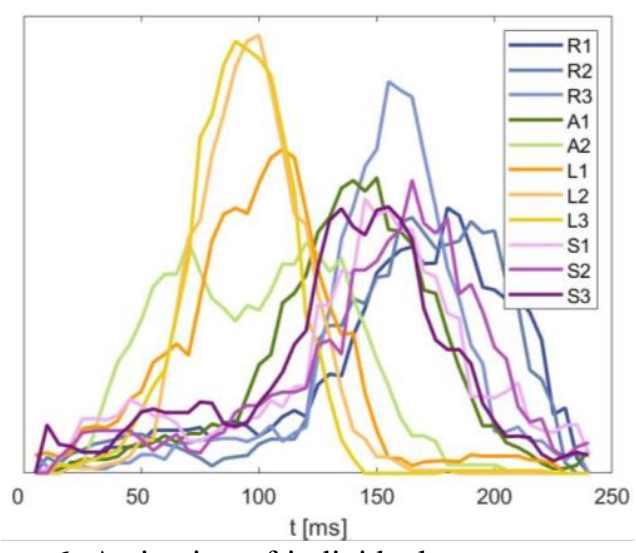

Figure 6. Activation of individual segments over time; RBBB record (the positions of the segments in the heart ventricle are shown in Figure 2).

After determining the center of gravity of each curve, the resulting activation map of the heart ventricle was created (Figure 7). The determined distribution of segment activation corresponds to the expected activation for the LBBB and RBBB records.

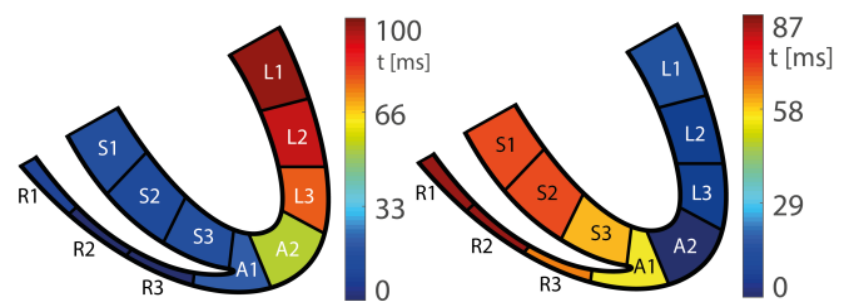

Figure 7. Heart ventricle activation map of the record with LBBB (left) and RBBB (right); segments R1, R2, and $\mathrm{R} 3$ are in the right ventricle; L1, L2, and L3 are in the left ventricle; S1, S2, and S3 are in the septum; A1 and $\mathrm{A} 2$ are in the apex.

\section{Conclusion}

A method for creating activation maps from only six standard precordial leads and knowledge of heart geometry was introduced. All calculated activation maps correspond to the theoretical expectation about the propagation of depolarization in LBBB and RBBB records. It is necessary to test the algorithm on data with known segment activation times for a more detailed evaluation. This data is not yet available.

The proposed algorithm can be useful for selecting the right therapy targeted individually for each patient. Although patients have the same diagnosis (for example, LBBB or RBBB), they do not always have the same sequence of activated segments. This method may also better describe the activation of heart in patients with nonspecific intraventricular conduction delay.

\section{Acknowledgments}

This work was supported by grant Czech Science Foundation with grant number GA17-13830S and by Internal grant Brno University of technology with grant number FEKT/FIT-J-19-5800.

\section{References}

[1] P. Jurak, U. C. Nguyen, I. Viscor, P. Andrla, F. Plesinger, F. W. Prinzen, J. Halamek, and P. Leinveber, "Epicardial Isochrones From a New High-Frequency ECG Imaging Technique," Computing in Cardiology 2018, vol. 45, Sept. 2018.

[2] M. Milanic, V. Jazbinsek, R. S. MacLeod, D. H. Brooks, and R. Hren, "Assessment of Regularization Techniques for Electrocardiographic Imaging," J Electrocardiol, vol. 47, no. 1, pp. 20-28, Feb. 2014.

[3] M. J. M. Cluitmans, R. L. M. Peeters, R. L. Westra, and P. G. A. Volders, "Noninvasive Reconstruction of Cardiac Electrical Activity: Update on Current Methods, Applications and Challenges," Neth Heart J, vol. 23, no. 6, pp. 301-311, Apr. 2015.

[4] L. R. Bear, Y. S. Dogrusoz, J. Svehlikova, J. Coll-Font, W. Good, E. van Dam, R. Macleod, E. Abell, R. Walton, R. Coronel, M. Haissaguerre, and R. Dubois, "Effects of ECG Signal Processing on the Inverse Problem of Electrocardiography," Computing in Cardiology 2018, vol. 45, Sept. 2018.

[5] P. Jurak, J. Halamek, J. Meluzin, F. Plesinger, T. Postranecka, J. Lipoldova, M. Novak, V. Vondra, I. Viscor, L. Soukup, P. Klimes, P. Vesely, J. Sumbera, K. Zeman, RS. Asirvatham, J. Tri, SJ. Asirvatham, P. Leinveber, "Ventricular Dyssynchrony Assessment Using Ultra-High Frequency ECG Technique", J Interv Card Electrophysiol, vol. 49, no. 3, pp. 245-254, 2017, doi:10.1007/s10840-0170268-0.

[6] P. Jurak, J. Halamek, P. Leinveber, V. Vondra, L. Soukup, P. Vesely, J. Sumbera, K. Zeman, L. Martinakova, T. Jurakova, and M. Novak, "Ultra-High-Frequency ECG Measurement," Computing in Cardiology 2013, vol. 40, pp. 783-786, Sept. 2013.

[7] F. Plesinger, J. Jurco, P. Jurak and J. Halamek, "Robust Multichannel QRS Detection," Computing in Cardiology 2014, vol. 41, pp. 557-560, Sept. 2014.

[8] F. Plesinger, J. Jurco, J. Halamek, P. Leinveber, T. Reichlova and P. Jurak, "Multichannel QRS Morphology Clustering - Data Preprocessing for Ultra-High-Frequency ECG Analysis," Cardiotechnix 2015, 2015.

Address for correspondence:

Radovan Smisek.

Department of Biomedical Engineering, Faculty of Electrical Engineering and Communication, Brno University of Technology

Technicka 3058/12, 61600 Brno, Czech Republic. smisek@feec.vutbr.cz 